nische Relevanz im Hinblick auf "harte" klinische Parameter bewiesen.

Im gleichen Sinn hatte eine kürzlich vorgelegte Metaanalyse keine Verbesserung hinsichtlich klinischer Parameter gezeigt. Protektionssysteme konnten hier nur in nachgeordneten Kategorien, aber nicht im
Hinblick auf Mortalität und Morbidität punkten (Pagnesi M et al. Int J Cardiol. 2016; 221:97-106).

Ein Grund, die TAVI aus neurologischer Sicht zu einer risikoärmeren Prozedur zu erklären, sind die bislang vorgelegten Daten zu Protektionssystemen jedenfalls noch nicht.

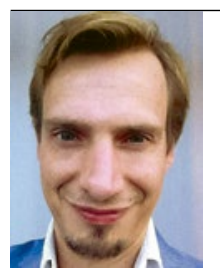

Dr. med.

Frank Stachulski

Abteilung Neurologie, Bundeswehrkrankenhaus Berlin

\title{
Hypertonie: Kochsalzzufuhr beeinflusst Effektivität von Spironolacton
}

\section{Kürzlich veröffentlichte Daten der Studie PATHWAY-2 haben die Aufmerksamkeit auf Spironolacton als Zusatztherapie bei therapie- resistenter Hypertonie gelenkt. Nun wurde nach Parametern gesucht, die mit einem Therapieerfolg durch Spironolacton bei resistenten Hypertonikern assoziiert sind.}

n einer retrospektiven Analyse wurden

79 Patienten mit resistenter Hypertonie untersucht. Die Therapieresistenz war definiert als ein Blutdruck von $\geq 140 / 90 \mathrm{mmHg}$ bei einer mindestens dreifachen Medikation unter Einschluss eines Diuretikums. Die Patienten erhielten zusätzlich zu ihrer bestehenden $\mathrm{Me}$ dikation 12,5-25 mg Spironolacton. Ein Ansprechen auf die Therapie wurde als systolische Blutdrucksenkung um mindestens $10 \mathrm{mmHg}$ definiert. Im Mittel erhielten die Patienten vier antihypertensive Medikamente.

Der Ausgangsblutdruck lag systolisch bei $153,6 \pm 22,3 \mathrm{mmHg}$. Spironolacton reduzierte den systolischen Blutdruck um 15,5 $\pm 20,7 \mathrm{mmHg}$. Diejenigen $\mathrm{Pa}$ tienten, die zu Beginn der Behandlung eine Natriumausscheidung von $>200$ mmol/ 24 Stunden hatten, zeigten eine signifikant höhere Blutdrucksenkung als Patienten mit einer Natriumausscheidung $<200 \mathrm{mmol} / 24$ Stunden $(\mathrm{p}=$ 0,008, Abb. 1).

Eine multivariate Analyse bestätigte die 24-Stunden-Natriumausscheidung im Urin als signifikanten Prädiktor der Blutdruckantwort $(p=0,021)$ nach Adjustierung für mögliche Einflussgrößen einschließlich eines primären Aldosteronismus.

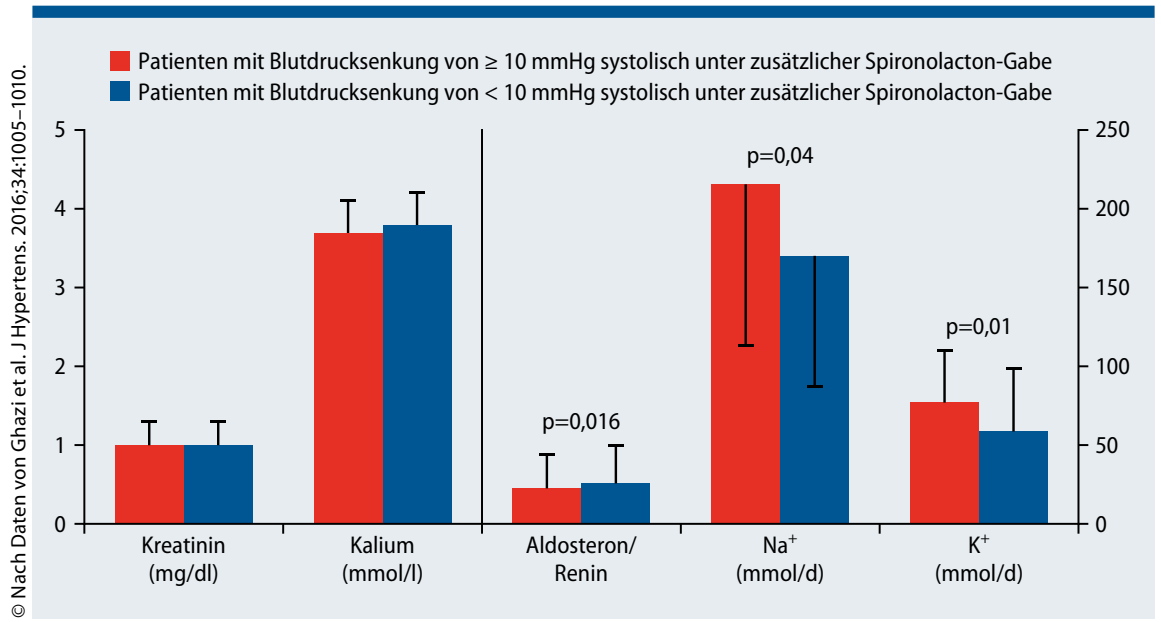

Abb. 1: Blutdrucksenkung bei therapieresistenten Hypertonikern unter zusätzlicher Gabe von Spironolacton.
Ghazi L et al. Urinary sodium excretion predicts blood pressure response to spironolactone in patients with resistant hypertension independent of aldosterone status. J Hypertens. 2016; 34:1005-1010.

\section{Kommentar}

In der Studie PATHWAY-2 wurden die Effekte von Spironolacton bei resistenter Hypertonie quantifiziert. Die Befunde ergaben, dass Spironolacton ein brauchbares zusätzliches Medikament bei resistenter Hypertonie ist. Die vorliegenden Daten zeigen nun, dass die Effektivität von Spironolacton offenbar mit der Kochsalzzufuhr der Patienten zusammenhängt: Wenn die Hypertoniker eine besonders hohe Kochsalzzufuhr aufweisen, ist Spironolacton besonders effektiv.

Dies zeigt, dass der Natriumhaushalt bei resistenter Hypertonie wahrscheinlich eine wichtige Rolle spielt. Das könnte über die vorliegenden Ergebnisse hinaus auch bedeuten, dass ein erster Schritt bei resistenter Hypertonie generell in der Steigerung der Natriurese bestehen könnte. Gerade bei resistenter Hypertonie ist nicht selten auch die Nierenfunktion eingeschränkt, was für die Anwendung von Spironolacton eine Limitation ist. In diesen Fällen sollten auch alternative Wege, die Natriumbilanz zu verbessern, erwogen werden, z.B. diätetisch oder durch intensivierte Diuretikatherapie.

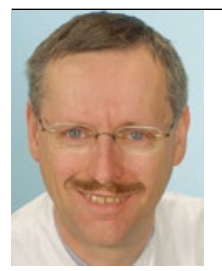

Prof. Dr. med. Walter Zidek Medizinische Klinik IV, Charité Universitätsmedizin Berlin 